\title{
Effect of a slowly resorbing cross-linked collagen membrane with highly soluble biphasic calcium phosphate on localized bone regeneration
}

\author{
You-Kyoung Kim*, Yinzhe An*, Jae-Kook Cha, Jung-Seok Lee, Ui-Won Jung and Seong-Ho Choi \\ Research Institute for Periodontal Regeneration, Department of Periodontology, College of Dentistry, \\ Yonsei University, Seoul, Korea
}

\section{Introduction}

The aim of this study was to evaluate bone regenerative capacity of a cross-linked porcine collagen membrane (CM, Collagen membrane$P \circledast$, Genoss, Suwon, South Korea) with highly soluble biphasic calcium phosphate (BCP, New Bone, Genoss, Suwon, South Korea).

\section{Materials and Methods}

Ten 16 weeks old New Zealand white male rabbits were used. Four circumferential bony defects were created on sagittal plane with a $8 \mathrm{~mm}$ diameter trephine bur under copious saline irrigation. Each defect was randomly allocated following 4 groups.

1) BCP-CM : Biphasic calcium phospate (HA/ $\beta$-TCP : 20/80) with cross-linked porcine collagen membrane. $0.1 \mathrm{~g}$ of $\mathrm{BCP}$ particles grafted defect was covered with porcine collagen membrane.

2) $\mathrm{CM}:$ The defect was covered with porcine collagen membrane without bone graft materials

3) BCP : The defect was filled with prepared $0.1 \mathrm{~g}$ of BCP particles.

4) Control : The defect was filled with blood clot.

Animals were sacrificed at 2 weeks $(n=5)$ and 8 weeks $(n=5)$.
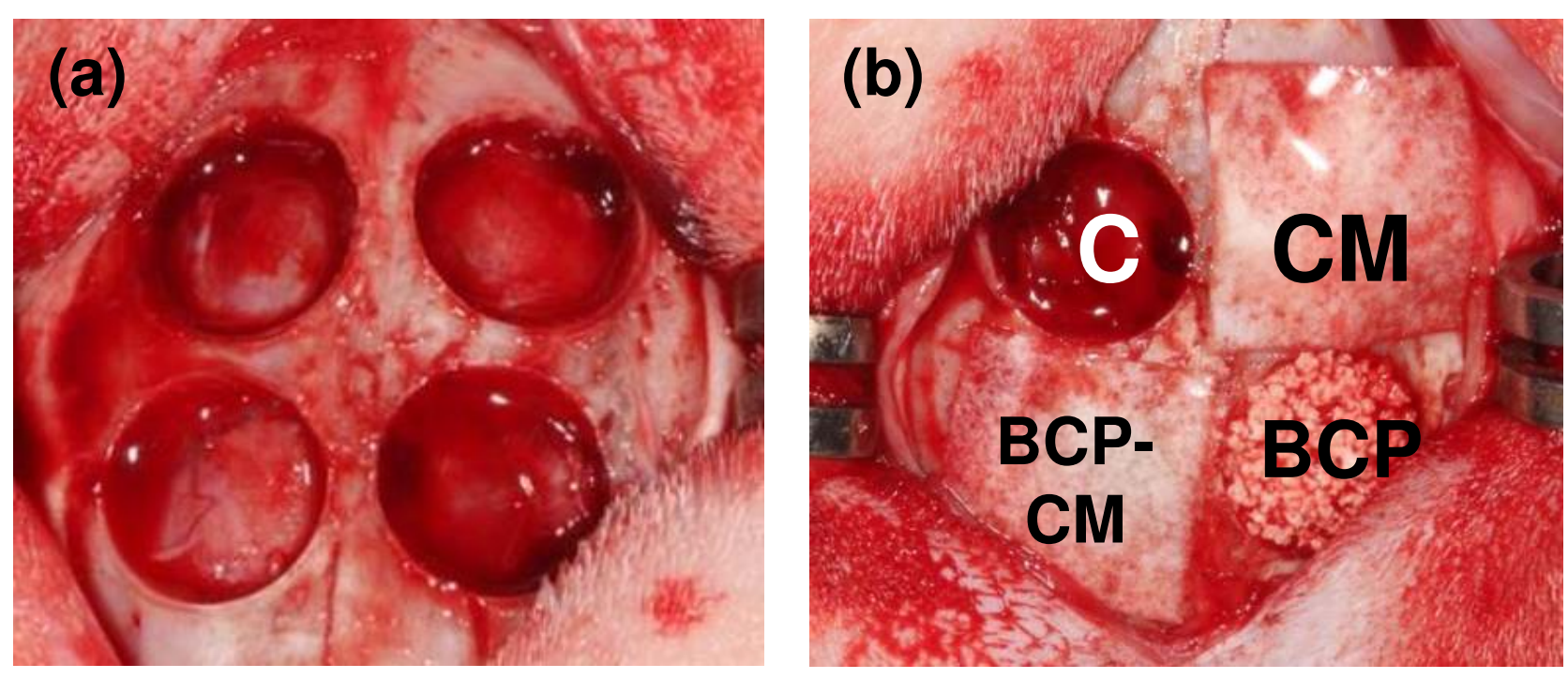

Figure1: Clinical photographs of the experimental sites. (a) Four circumferential defects with $8 \mathrm{~mm}$ diameter were created on ten New Zealand white rabbits' calvaria. Full thickness of the bone was carefully removed. (b) Four different groups were randomly allocated. $(\mathrm{C}$ : control, $\mathrm{CM}$ : cross-linked porcine collagen membrane, $\mathrm{BCP}$ : $\mathrm{BCP}$ with HA/ $\beta$-TCP: $20 / 80$, BCP-CM : BCP with HA/ $\beta$-TCP: $20 / 80$ and covered with cross-linked porcine collagen membrane.)

\section{Acknowledgments}

This study was supported by a grant of the Korea Health technology R\&D Project, Ministry of Health \& Welfare, Republic of Korea
Results

\begin{tabular}{|c|c|c|}
\hline & 2 weeks & $\mathbf{8}$ weeks \\
\hline Control & $7.40 \pm 2.21$ & $8.20 \pm 1.46$ \\
\hline CM & $11.90 \pm 2.20$ & $9.01 \pm 3.52$ \\
\hline BCP & $21.97 \pm 2.27^{\mathrm{a}}$ & $22.48 \pm 1.10^{\mathrm{a}}$ \\
\hline BCP-CM & $27.12 \pm 3.99^{\mathrm{a}, \mathrm{b}}$ & $25.75 \pm 1.82^{\mathrm{a}, \mathrm{b}}$ \\
\hline
\end{tabular}

Table 1: Area of total augmentation (mm2; Group Mean \pm SD; $n=5)$ a : Significantly different from control group at 2 and 8 weeks postoperative $(\mathrm{p}<0.05)$.

$\mathrm{b}$ : Significant difference between BCP group and BCP-CM group at same experimental period $(\mathrm{p}<0.05)$

Within the augmented area, a significantly higher bone formation was observed for the two BCP groups $\left(5.35 \pm 2.42 \mathrm{~mm}^{2} ; 3.91 \pm 1.47 \mathrm{~mm}^{2}\right)$ (BCP-CM, BCP) compared to CM $\left(1.89 \pm 0.82 \mathrm{~mm}^{2}\right)$ and control $\left(1.79 \pm 1.18 \mathrm{~mm}^{2}\right)$ at 2 weeks. Similar observations were made at 8 weeks being significantly different between two BCP groups (BCP$\mathrm{CM}: 7.33 \pm 1.90 \mathrm{~mm}^{2}$; BCP: $\left.7.71 \pm 1.26 \mathrm{~mm}^{2}\right)$ and other groups $(\mathrm{CM}$ : $2.10 \pm 1.03 \mathrm{~mm}^{2} ; \quad$ C: $\left.2.97 \pm 0.95 \mathrm{~mm}^{2}\right)$. Bone formation increased significantly from 2 to 8 weeks in groups BCP-CM and BCP only.

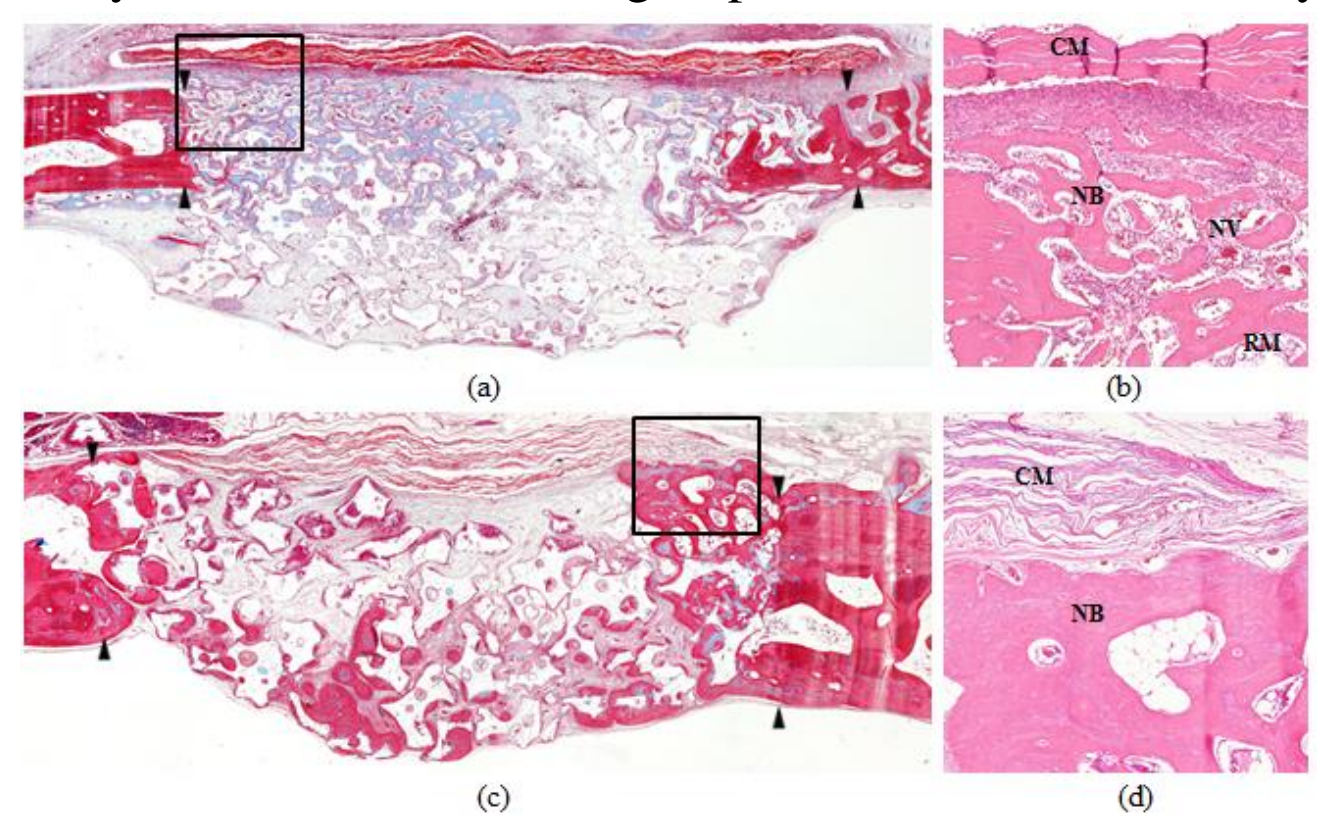

Figure 2 : Transversal histologic section of BCP-CM group at 2 weeks ( $a$ and $b$ ) and 8 weeks ( $c$ and d). Arrow head: defect margin, NB: new bone, AC: adipocyte, RM: residual material, NV: new vessel

\section{Conclusion}

A slowly resorbing cross-linked porcine collagen membrane combined with highly soluble BCP enhances regeneration, providing sufficient space maintaining capacity to supplement rapid resorption of $\mathrm{BCP}$ which has superior osteoconductivity. 